\title{
Newsfronts
}

\section{Fish and Blood: New Techniques to Study Hematopoiesis}

A recent study in zebrafish presents a potentially important new system for studying the process of stem cell biology and hematopoiesis, the process by which blood is produced.

The zebrafish has proved to be a valuable model for studying hematopoiesis. This species is ideal for large-scale mutagenesis studies, because considerable numbers of zebrafish can be raised and maintained simultaneously. Furthermore, the mature blood cells produced by the adult zebrafish closely resemble their mammalian counterparts, and arise from a process very similar to the hematopoietic pathway seen in mammals. Finally, zebrafish embryos are transparent, allowing for easy observation of blood formation.

Researchers have previously identified dozens of hematopoietic mutants in the zebrafish, although many of these mutations produce similar phenotypes or result in embryonic lethality for homozygotes, and thus remain poorly characterized. In an effort to establish better methods for studying such zebrafish mutants, a group of researchers led by Leonard Zon from the Children's Hospital Boston (Boston, MA) developed a technique for transplantation of hematopoietic stem cells derived from whole-kidney marrow (WKM), which is the zebrafish equivalent of mammalian bone marrow and the source of all blood cell production.

Zon's group began by developing a system for characterizing the relative proportions of different cell types in wild-type WKM, using flow cytometry to sort blood cells from a variety of fish lines expressing green fluorescent protein (GFP) from different blood cell-specific promoters; they then compared these findings to WKM derived from different anemic mutant fish lines (Nat. Immunol., December 2003). Their findings indicated that several of these mutations were lethal to embryos as a result of defects that blocked the maturation of precursor cells for certain blood cell lineages, a phenotype that was mildly apparent even in seemingly normal heterozygotes.

Zon and his colleagues developed a transgenic line expressing GFP from an erythroid-specific promoter, and transplanted WKM thus labeled into moonshine $(\mathrm{mon} / \mathrm{mon})$ and vlad tepes $\left(\right.$ gatal $\left.^{-/-}\right)$ mutants, lines that normally die by 14 days after fertilization. In both lines, there was rescue of normal blood production, and the transparency of the embryos allowed the researchers to visualize easily the migration and growth of the labeled cells.

The researchers also generated doubletransgenic zebrafish, which produced GFPtagged leukocytes and erythroid cells labeled with dsRED; after transplantation of WKM from this transgenic line, the researchers could easily track the migration of the two cell types. They found that the dsRED+ cells remained largely in circulation, while the GFP+ cells achieved two different fates, forming both lymphocytes and myelomonocytic cells, both of which could be easily identified by their visual characteristics.

According to Zon, this work marks two important achievements: the ability to sort blood cell types easily by flow cytometry, and the technique for marrow transplantation. He tells Lab Animal, "This work establishes the basis for a genetic approach for studying marrow homing, engraftment, proliferation, and differentiation." Given the advantages of zebrafish embryos as a mutant model, "it is [now] possible to study individual genes and their role in short-term and long-term reconstitution." $\mathrm{He}$ indicates that also on the horizon are additional transplantation and gene expression profiling experiments, which they anticipate will yield yet more insights into the genetics of stem cell biology and hematopoiesis.

- Michael Eisenstein

\section{Pig News in Transgenics}

Using a virus related to HIV as a vector, a group of German scientists has developed a highly efficient method for creating transgenic pigs, thus overcoming a major obstacle to the use of these animals not only as research models, but also for the production of therapeutic proteins and organs for xenotransplantation.

Traditional transgenesis by microinjection of DNA into the pronuclei of fertilized eggs is extremely inefficient, with a success rate of less than $3 \%$ in rodents and even lower in farm animal species. These low efficiency rates correlate directly with high production costs, thus hampering the production of transgenic swine.

Viral transgenesis using retroviral vectors has met with limited success, but researchers have recently used lentiviruses-members of a family of complex retroviruses-to create transgenic mice. Lentiviruses infect both dividing and nondividing cells, infect a broad variety of cells, and transduce murine and human embryonic stem cells and preimplantation embryos.

Now, a group led by Eckhard Wolf and Alexander Pfeifer of the LudwigMaximilians University in Munich, Germany, report using a lentiviral vector carrying the green fluorescent protein (GFP) transgene and a ubiquitous promoter to produce transgenic swine (EMBO Rep., November 2003). They injected the vector into single-cell embryos and implanted these into recipient females. Of the 46 piglets born, $32(70 \%)$ carried the transgene, and 30 (94\%) of these showed GFP expression in all tissues. The group also demonstrated that the transgene was transmitted through the germline and that a tissue-specific promoter could be used to limit the expression of the transgene to specific tissues.

According to Pfeifer, the group will now attempt to generate strains of pigs carrying transgenes relevant to xenotransplantation. He tells Lab Animal, "The combination of lentiviral transgenesis and RNAi technology should allow for the expression of foreign genes as well as the knockdown of porcine genes from one and the same vector."

-Tanja Schub

("Newsfronts" continues on p. 10) 


\section{News Updates Spina Bifida Gene Identified}

Australian researchers have identified a gene that causes neural tube disorders (NTDs) in mice, a finding that may lead to genetic testing and new treatments for the conditions in humans.

The NTDs spina bifida and anencephaly are the second most common type of birth defect, affecting 1 in 1,000 births, and are known to have both genetic and environmental components. Women who take in sufficient folate at the time of conception have a substantially reduced risk of delivering a child with a NTD; however, $30 \%$ of NTDs seem to be folate-resistant.

The mutant mouse curly tail $(c t / c t)$ has long been used as a model for studying NTDs, although the causative mutation was not known. Now, a group led by Stephen M. Jane of the Royal Melbourne Hospital (Victoria, Australia) report that 100\% of mice lacking a functional copy of the gene Grhl3 exhibited a phenotype consistent with severe spina bifida (Nat. Med., December 2003). Chances seem good that this gene, which encodes a developmental transcription factor, will be found to be involved in spina bifida in humans, because the mouse and human genes are virtually identical.

\section{With a Little Help from Their Friends}

Everybody knows how helpful the support of a few good friends can be, but a new study in baboons goes a step farther, providing evidence that strong social ties can directly benefit reproductive fitness by enhancing the likelihood of infant survival.

The study, conducted by Joan B. Silk from the University of California at Los Angeles, incorporated data collected from two well-studied groups of wild savannah baboons in Kenya (Science, 14 November 2003). The data indicated a direct positive correlation between the level of socialization exhibited by female baboons, as indicated by the frequency with which subjects were found in close proximity to or engaged in grooming with other females, and the probability of their offspring surviving to one year of age. These apparent benefits of increased social contact remained statistically significant even after the data were adjusted to account for differences in social standing and habitat conditions.

Previous research has identified similar benefits resulting from regular social contact in a variety of nonhuman primate and other mammalian species. Humans also show such beneficial effects, and the authors cite several studies that indicate the positive impact of strong social ties on health and well-being. Although the specific mechanisms behind this increased infant survival remain to be delineated, this study provides new evidence of the important adaptive value of social relationships.

\section{A Shared Switch for Different Drugs}

New research has identified a single protein that seems to be involved in the signaling cascades for three different types of psychotropic drug.

d-amphetamine, LSD, and PCP all fall under the generic classification of psychotomimetics - that is, compounds that can induce mental symptoms similar to those seen in schizophrenic patients. These three compounds act specifically on dopaminergic, serotonergic, and glutaminergic neurons, respectively-the same three subtypes of neurons whose dysfunction has been implicated in the etiology of schizophrenia. In an effort to identify a common element for these three pathways, a group led by Paul Greengard from Rockefeller University (New York, NY) examined the potential role played by DARPP-32, a key signaling regulator expressed in all three neuronal subtypes (Science, 21 November 2003).

Transgenic mice lacking expression of DARPP-32 showed an altered response to all three drugs and showed a significant attenuation of the stereotypical behaviors, such as increased repetitive motion, that are observed in similarly treated wild-type mice. Treatment with any of these drugs was also found to trigger the phosphorylation of specific serine and threonine residues on DARPP-32 in the striatum and frontal cortex, regions of the brain that are dysfunctional in schizophrenic patients. By comparison, treatment did not trigger phosphorylation of other unrelated signaling proteins, nor did it affect DARPP-32 phosphorylation in other structures of the brain. By mutating individual serine and threonine residues, Greengard's group was also able to identify the specific amino acids that are involved in mediating the action of DARPP-32 in response to each drug. These combined findings strongly support a model in which DARPP-32 is a shared regulatory component for signaling in response to a variety of psychotomimetic agents. 